\title{
VULNERABILIDADE NO DESENVOLVIMENTO DA CRIANÇA: INFLUÊNCIA DA JUVENTUDE E CONDIÇÕES DE SAÚDE MATERNAS*
}

Daniel Ignacio da Silva1, Verônica de Azevedo Mazza²

'Enfermeiro. Mestre em Enfermagem. Prefeitura Municipal de Colombo. Colombo-PR-Brasil. ${ }^{2}$ Enfermeira. Doutora em Enfermagem. Universidade Federal do Paraná. Curitiba-PR-Brasil.

RESUMO: Pesquisa exploratória qualitativa que objetivou conhecer a compreensão do enfermeiro sobre a vulnerabilidade no desenvolvimento da criança. Foi realizada com 39 enfermeiros atuantes em Unidades Municipais de Saúde com Estratégia Saúde da Família em Curitiba, Paraná, Brasil. Para a coleta de dados utilizouse a entrevista semiestruturada, e para análise a hermenêutica dialética, sendo respeitados os princípios éticos. Os resultados apontaram que o enfermeiro compreende a juventude e as condições da saúde materna como situações adversas ao desenvolvimento infantil e assinalam a necessidade deste aperfeiçoar suas tecnologias de cuidado que promovam a saúde das gestantes adolescentes, incentivem apropriadamente a alimentação infantil e articulem uma adequada rede social de apoio às mães que se encontram em situação de adoecimento. A compreensão da vulnerabilidade no cuidado à criança permite a reorientação do modelo assistencial, que fundamentado nos princípios de integralidade e equidade, seja direcionado às necessidades da criança e sua família. DESCRITORES: Vulnerabilidade em saúde; Desenvolvimento infantil; Adolescente; Relações mãe-Filho; Enfermagem.

\section{VULNERABILITY IN CHILD DEVELOPMENT: THE INFLUENCE OF THE MOTHER'S YOUTH AND HEALTH CONDITIONS}

\begin{abstract}
This exploratory qualitative research aimed to investigate nurses' understanding regarding vulnerability in child development. It was undertaken with 39 nurses working in Municipal Health Centers with the Family Health Strategy in Curitiba, Paraná, Brazil. A semistructured interview was used for data collection, and dialectical hermeneutics for analysis, ethical principles being respected. The results indicated that nurses understand the mother's youth and health conditions as situations which are adverse to child development, and indicate the need for nurses to improve their care technologies which promote the health of teenage mothers, appropriately encourage breastfeeding and articulate an adequate network of social support for those mothers who are ill. The understanding of vulnerability in the care for the child allows the reorientation of the care model which, based in the principles of comprehensiveness and equity, may be directed at the needs of the child and her family. DESCRIPTORS: Vulnerability in health; Child development; Adolescent; Mother-Child relationships; Nursing.
\end{abstract}

\section{VULNERABILIDAD EN EL DESARROLLO DEL NIÑO: INFLUENCIA DE LA JUVENTUD Y CONDICIONES DE SALUD MATERNAS}

RESUMEN: Investigación exploratoria cualitativa cuyo objetivo fue conocer la comprensión del enfermero acerca de la vulnerabilidad en el desarrollo del niño. Fue realizada con 39 enfermeros actuantes en Unidades Municipales de Salud con Estrategia Salud de la Familia en Curitiba, Paraná, Brasil. Los datos fueron obtenidos por entrevista semiestructurada, y para el análisis fue utilizada la hermenéutica dialética, siendo respetados los princípios éticos. Los resultados apuntan que el enfermero comprende la juventude y las condiciones de la salud materna como situaciones adversas al desarrollo infantil e indican la necesidad de perfeccionarse tecnologías de cuidado para promover salud de las gestantes adolescentes, incentivar adecuadamente la alimentación infantil y articular una apropiada red social de apoyo a las madres que están en situación de enfermedad. La comprensión de la vulnerabilidad en el cuidado del niño posibilita la reorientación del modelo asistencial, lo cual sea direccionado a las necesidades del niño y de su familia fundamentándose en los principios de integralidad y equidad. DESCRIPTORES: Vulnerabilidad en salud; Desarrollo infantil; Adolescente; Relaciones madre-hijo; Enfermería.

*Artigo originado da dissertação "Vulnerabilidade no desenvolvimento da criança segundo o enfermeiro da Estratégia Saúde da Família" apresentada ao Programa de Pós-Graduação em Enfermagem da Universidade Federal do Paraná, 2012.

Recebido: 05/04/2013 Finalizado: 09/04/2014

\section{Autor Correspondente:}

Daniel Ignacio da Silva

Prefeitura Municipal de Colombo

Rua José Maria da Silva Paranhos, 513 - 83409-470 Colombo-PR-Brasil

E-mail: daniel.silva1076@usp.br 


\section{INTRODUÇÃO}

O desenvolvimento infantil pode ser entendido como um processo vital que resulta da interação entre os fenômenos de crescimento, maturação e aprendizagem; no qual ocorrem mudanças qualitativas nas funções do indivíduo, que podem ser percebidas em suas habilidades e comportamentos nos âmbitos físico, intelectual, emocional e social ${ }^{(1)}$.

O desenvolvimento da criança, durante a sua primeira infância ( 0 aos 3 anos), está condicionado à aquisição de habilidades e competências particulares de cada etapa da vida da $\operatorname{criança}^{(2)}$, por isso, mostra-se necessário o atendimento das suas necessidades essenciais, que são aquelas que propiciam mecanismos fundamentais para a obtenção dos potenciais intelectual, social, emocional e físico ${ }^{(3)}$. Estas necessidades descritas estão relacionadas à sobrevivência e ao desenvolvimento integral do indivíduo, independente de sua etnia, classe social, condições físicas e mentais e o não provimento dessas pode prejudicar o desenvolvimento da criança ${ }^{(4)}$.

Existem evidências de que na primeira infância, a criança pode experienciar situações adversas, que conforme a neurociência, biologia molecular, epigenética e ciências comportamentais e sociais ${ }^{(4-5)}$, influenciarão seu êxito educacional, suas habilidades emocionais, suas condições de saúde ao longo de sua vida, sua capacidade produtiva e sua cidadania ${ }^{(6)}$. A exposição da criança à adversidade significativa pode produzir ativação demasiada de sistemas de resposta ao estresse, incluindo os hormônios do estresse, como o cortisol, que podem acarretar prejuízos ao desenvolvimento cerebral( ${ }^{(4)}$.

Sendo assim, é preciso compreender a criança como um sujeito social com características e necessidades próprias, reconhecendo que o cuidado prestado às crianças tem papel decisivo no desenvolvimento de suas potencialidades ${ }^{(7)}$. O que mostra que este cuidado deve ser provido de forma integral, considerando as condições psicossociais e biológicas da criança, conforme o seu grau de vulnerabilidade ${ }^{(8)}$.

A vulnerabilidade no desenvolvimento da criança pode ser definida como a chance ou oportunidade da criança sofrer prejuízos ou atrasos em seu desenvolvimento devido à influência de aspectos de ordem individual, social e programática, que são denominados situações adversas ${ }^{(9)}$. Assim, a complexidade da vulnerabilidade demanda intervenções de natureza multi e interdisciplinar, que devem ser desenvolvidas com diversos subsídios teóricometodológicos ${ }^{(10)}$. A aplicação do conceito de vulnerabilidade no cuidado à criança permite a reorientação do modelo assistencial, e a superação do conceito biologista e fragmentado de desenvolvimento infantil, permitindo a operacionalização do conceito de integralidade nas práticas assistenciais ${ }^{(9)}$.

A dimensão individual da vulnerabilidade no desenvolvimento da criança é composta de aspectos biológicos, comportamentais e afetivos, podendo ter prejuízos no seu desenvolvimento devido a fragilidades nas relações sustentadoras contínuas e falta de proteção física e segurança ${ }^{(3,9)}$.

Entre estes aspectos descritos, destaca-se que as condições maternas podem influenciar o desenvolvimento infantil. Entre estas, a juventude materna, a gravidez na adolescência tem sido considerada uma condição que pode trazer agravos à saúde, tanto da criança como da mãe ${ }^{(11)}$. Estudos apontam que as adolescentes aderem menos ao pré-natal e têm mais dificuldade para receber orientações com relação aos cuidados pessoais e do recém-nascido, aumentando a vulnerabilidade que envolve esse grupo ${ }^{(11-12)}$.

As condições de saúde materna são salientadas, pois existem evidências que a criança pode ser exposta a situações de vulnerabilidade, caso seus cuidadores não estejam com saúde mental ou emocional adequada, podendo haver situação de desajustes familiares seguidos de desestruturação de seus membros ${ }^{(13)}$.

Neste sentido, este estudo pode contribuir para a utilização da concepção de vulnerabilidade como subsídio teórico para a construção de intervenções de Enfermagem que promovam o desenvolvimento infantil, que permitam ao enfermeiro identificar, em sua práxis de cuidado, as crianças que forem privadas de suas necessidades básicas e que se encontram diante de situações que prejudicarão o seu desenvolvimento.

Diante disto, o objetivo do estudo foi conhecer a compreensão do enfermeiro sobre a vulnerabilidade no desenvolvimento da criança. 


\section{MÉTODO}

Trata-se de uma pesquisa exploratória qualitativa realizada em 39 Unidades Municipais de Saúde (UMS) com Estratégia Saúde da Família (ESF), localizadas em distritos sanitários da cidade de Curitiba-PR-Brasil, que apresentavam concomitantemente os resultados de Indicadores de Inserção Social (IIS) e de Indicadores de Qualidade do Domicílio (IQD) iguais ou inferiores à média do Município ${ }^{(7)}$. Os distritos sanitários escolhidos conforme os critérios acima estabelecidos foram: Cajuru, Boqueirão, Bairro Novo, Pinheirinho e CIC (Cidade Industrial de Curitiba).

Para convidar os sujeitos, o pesquisador solicitou que a coordenação da UMS indicasse, mediante o planejamento local de saúde, a área de maior risco social e epidemiológico da unidade. Logo, os participantes da pesquisa foram 39 enfermeiros que cumpriram os seguintes critérios de inclusão: atuar nas equipes das áreas de maior risco das UMS, ter dois anos completos de atuação na ESF, além de concordar e assinar o Termo de Consentimento Livre e Esclarecido. Foram excluídos da amostra os enfermeiros que não cumpriram os critérios de inclusão, e os que estavam de férias, licença médica ou em afastamento por outro motivo.

A coleta dos dados ocorreu entre fevereiro e março de 2012, por meio de entrevistas individuais semiestruturadas em que foram tratados os seguintes temas: aspectos prejudiciais ao desenvolvimento da criança em sua primeira infância, situações de prejuízos ao desenvolvimento infantil vivenciadas pelo enfermeiro, e ações de promoção ao desenvolvimento infantil praticadas pelo enfermeiro ${ }^{(14)}$.

A análise das falas dos enfermeiros foi realizada por meio da hermenêutica dialética, e com base na matriz de análise da vulnerabilidade da criança diante de situações adversas ao seu desenvolvimento ${ }^{(9,14)}$. Esta matriz permitiu uma síntese da situação da criança e de sua família e uma aproximação, com este fenômeno na práxis assistencial dos profissionais, que mostrasse como este conceito era compreendido em suas vivências diante dos aspectos biológicos, comportamentais, afetivos, contextuais, econômicos, sociais e político-programáticos. Com a interpretação das falas dos sujeitos, emergiram as categorias Juventude Materna e Condições da Saúde Materna, na dimensão individual da vulnerabilidade.

As unidades de registro foram apresentadas e identificadas com a abreviação (E) e numeradas sequencialmente, garantindo o anonimato dos respondentes. Esta pesquisa obteve a aprovação do Comitê de Ética do Setor de Ciências da Saúde da Universidade Federal do Paraná, pelo registro n. 1170.095.11.6.

\section{RESULTADOS}

\section{Juventude Materna}

Nesta categoria, o enfermeiro compreende a juventude materna como uma possível situação adversa para o desenvolvimento da criança, pois esta condição influencia o cuidado à criança, e a formação de vínculos afetivos de seus cuidadores para com a criança:

A maioria [...] são adolescentes nessa área. Por que assim, vamos começar pela amamentação que seria um suporte para criança [...] mas a maioria não amamenta, acaba deixando de amamentar e não dá os cuidados básicos de higiene. (E4)

Tem bastante pais adolescentes na nossa área [...] isso influencia, porque às vezes, eles não têm aquele cuidado que realmente a criança precisa [...] os cuidados básicos [...] nem de higiene, nem de moradia [...]. (E10)

A visão que eu tenho quando faço o pré-natal de muitas gestantes aqui é que são gestantes muito jovens [com] treze, doze, quatorze anos. A grande maioria sem nenhuma perspectiva de vida para o futuro por que [...] são meninas que não tem mais sonho de vida [...] tendo em vista que as crianças [...] que nasceram dessas mães já adolescentes também [...] não tem tanto cuidado com os filhos. Elas são jovens, às vezes não queriam ser mães [...]. (E15)

Eu acredito que as mães são muito novas. Doze, quatorze, quinze anos, então são crianças, que 
acabam tendo que cuidar de crianças ou, acabam abandonando com as avós ou deixando o dia inteiro na escola, então não tem experiência para estar assumindo a maternidade e nem responsabilidade [...] muitas delas acabam criando, sozinha sem um companheiro, porque o que uma criança de dezessete, quinze anos sabe sobre querer formar uma família [...] a menina, com o filho pequeno, às vezes tem que trabalhar, não trabalha fica em casa só para cuidar do filho, a avó acaba sustentando aquela situação. (E28)

A gente tem aqui na unidade, muitos casos de meninas de quatorze, quinze anos que tiveram gravidez devido um relacionamento de uma noitada, furtivo. São crianças que não são programadas, muitas vezes, vítimas de tentativas de aborto mal sucedidas, de mães que não estavam esperando, são adolescentes criando um bebê, brincando de boneca, com aquela criança e para você criar um vínculo afetivo, desta adolescente que está no momento dela, de imaturidade, é muito difícil. (E30)

\section{Condições da Saúde Materna}

Por outro lado, o enfermeiro compreende as condições de saúde das jovens mães, principalmente as questões de saúde mental, as quais podem prejudicar a construção dos vínculos familiares, os estímulos ao desenvolvimento infantil e o monitoramento das atividades da criança:

A situação da mãe, situação física materna da mãe, psicológica. Então uma mãe desnutrida, uma mãe obesa, mãe com alguma comorbidade e a situação psíquica da mãe também, emocional, às vezes é uma mãe depressiva [...] as questões relacionadas com, depois com a criança mesmo. (E19)

Ela tem uma história de esquizofrenia. Foi uma gravidez bem problemática, ela estava até no momento de descompensação da doença mental [...] é uma criança que até hoje assim não se expressa livremente, então, não é uma criança que fala, ela já está aproximadamente com uns dois anos e meio, mas é uma criança que ainda não fala espontaneamente comparando com as outras crianças da mesma idade. (E19)
A mãe tem problema sério de saúde, e ela não consegue acompanhar esta criança até a escola. Ela não sabe se ela foi, se ela não foi, às vezes a escola chama e ela não consegue ir, ela tem uma dificuldade bem grande de monitoramento [da criança]. (E3)

\section{DISCUSSÃO}

Na categoria Juventude Materna, o enfermeiro refere à situação da mãe adolescente, que em muitas ocasiões se encontram desamparadas sem o apoio necessário para o cuidado da criança. Deve-se considerar que a gravidez na adolescência não consiste somente em um ato biológico reprodutivo, mas em um processo social complexo, condicionado por aspectos individuais, sociais e econômicos, que afeta tanto as jovens, como seus familiares ${ }^{(11-12)}$.

Alguns estudos sugerem que a gravidez na adolescência tem reflexos de um complexo conjunto de situações como relações sexuais desprotegidas e com múltiplos parceiros sexuais, envolvimento com violência, insucesso escolar, depressão, crenças e atitudes que fortaleçam seu comportamento sexual de risco. Por outro lado, deve-se considerar que as normas sociais, nas quais essas adolescentes vivem, a pobreza e a falta de acesso a serviços de saúde também influenciam na prevalência de gravidez entre esse público específico ${ }^{(11-12)}$.

A gravidez na adolescência tem sido motivo de preocupação para gestores e autoridades. Atualmente, a gravidez tem sido prevalente em cerca de $25 \%$ das jovens entre 10 e 19 anos, estando porém, em Curitiba, local do estudo, em cerca de $14 \%$ das gestações ${ }^{(15)}$. A situação dessas adolescentes está relacionada, em grande parte, pela antecipação da vida sexual ativa dos adolescentes e pelas questões de classe social e etnia, sendo maior entre as negras ${ }^{(12,16)}$.

As adolescentes têm sofrido a discriminação gerada pelas desigualdades de gênero construídas historicamente, as quais estabelecem uma condição de inferioridade para a mulher(16). Estas mães são, em sua maioria, jovens na faixa etária entre 15 e 20 anos, solteiras, com dificuldades financeiras, sem apoio de seus parceiros, de suas famílias e do Estado, e as que mais abandonam seus filhos ${ }^{(17)}$. 
Por isso mostra-se necessário que a adolescência receba atenção especial das autoridades quanto à melhoria da qualidade educacional e da inclusão profissional dessas jovens mulheres por meio de políticas que busquem melhorar suas condições de vida ${ }^{(12,18)}$. Esta deve ter especial tratamento por parte das autoridades, principalmente quanto ao incremento de garantia do acesso das adolescentes a uma educação básica de qualidade que desenvolva a capacidade da adolescente em se prevenir de gravidezes indesejadas, além de permitir que esta tenha condições de entrar no mercado de trabalho e possa ter uma vida com dignidade cidadã $\tilde{}^{(18)}$.

O enfermeiro identifica a juventude da mãe como elemento que influencia o desmame precoce e os tipos de alimentos a serem oferecidos à criança. A literatura recomenda que até os seis meses de vida, o leite materno deve ser a única fonte de alimentos da criança, pois possui capacidade oferecer aporte proteicocalórico adequado, favorecendo sua proteção contra doenças e o seu desenvolvimento ${ }^{(19-20)}$.

Um estudo mostrou que a prevalência de aleitamento exclusivo tem sido menor entre adolescentes, e o desmame completo no final do terceiro mês também tem sido maior entre esse grupo $^{(21)}$. Outro estudo evidenciou que o desmame geralmente acontece dentro das primeiras semanas após o nascimento, o período crítico, em que muitas mães acham seu leite insuficiente, tomam medicações ou experienciam traumas mamilares ou dor mamária ${ }^{(19)}$.

Os resultados corroboram com um estudo que mostrou que, apesar dos benefícios da amamentação para a mãe, filho, e sociedade estarem bem evidenciados em pesquisas, ainda a prevalência de aleitamento materno permanece abaixo das metas mundiais, sendo que $75 \%$ das mulheres amamentam na alta hospitalar e somente $50 \%$ o fazem seis meses após o parto ${ }^{(19)}$. Outro estudo mostrou que entre as mulheres australianas, mais de $80 \%$ delas iniciam o aleitamento materno após o nascimento, contudo, menos de metade das crianças recebem leite materno aos seis meses ${ }^{(20)}$.

Tratando dos efeitos do desmame precoce, uma pesquisa concluiu que a sua prática, antes dos quatro meses, foi associada com o aumento da taxa de morbidade com o risco da descontinuidade da lactação; estando relacionado com a possibilidade de imaturidade em alguns órgãos como intestinos, rins e sistema imunológico, assim como com baixa exposição aos efeitos protetores do leite materno ${ }^{(22)}$. Por outro lado, outra pesquisa identificou que o atraso para a introdução de alimentos sólidos para além da idade de seis meses tem sido associado com um risco aumentado de desnutrição e a anemia ferropriva ${ }^{(23)}$.

Na categoria Condições da Saúde Materna, o enfermeiro descreve a situação do estado psicoemocional da mãe, como elemento que influencia a construção dos vínculos familiares. A mãe tem sido descrita, historicamente como a principal personagem para o cuidado infantil, e como a principal responsável pelo estímulo do desenvolvimento da criança ${ }^{(3)}$.

O enfermeiro alude sobre as dificuldades da mãe doente em estimular o desenvolvimento de seu filho. Um estudo estabeleceu que, em muitos casos, a mãe que sofre com doenças mentais como a depressão pós-parto, pode ter limitações para propiciar o desenvolvimento emocional e comportamental das crianças ${ }^{(24)}$.

Estes resultados estão de acordo com algumas pesquisas que evidenciaram que as mães deprimidas demonstram dificuldade de sensibilidade, respostas contingentes e modulação de estimulação ambiental para com seus filhos, sugestivas de mecanismos sociais e interacionais ${ }^{(13,24)}$. E embora haja esse sofrimento, muitas mães se esforçam para cuidar de seus filhos, independentemente do apoio social que tenham recebido ${ }^{(25)}$.

De outra forma, o enfermeiro expõe a influência da doença materna sobre o monitoramento da criança em suas atividades. Isto se relaciona com as experiências de aprendizado que a criança necessita desenvolver com sua família ou rede social, a partir do afeto que esta recebe de seus cuidadores. Todos os limites e estruturas necessitam ser iniciados com afeição e preocupação, pois é a partir destas que a criança construirá sua visão do mundo em que vive, e as relações com as pessoas ao seu redor ${ }^{(3)}$.

Este dado está de acordo com um estudo que mostrou que as fragilidades das relações entre pais e filhos podem comprometer o desenvolvimento da criança, se esta crescer sem o estabelecimento 
de limites, podendo apresentar dificuldades em algumas situações de sua vida ${ }^{(26)}$.

Portanto, a rede social de apoio para mães que se encontram com sua saúde debilitada, pode contribuir para o cuidado da criança com vistas à promoção do desenvolvimento infantil. Pois tem havido lacunas em grande parte das situações, ficando a criança, muitas vezes, sem o cuidado adequado, pela falta de vagas em creches ou instituições socioeducativas que poderiam auxiliá-las na proteção infantil(27). Embora, a família seja socialmente identificada como provedora dos cuidados à criança, é necessário que esta possua apoio das pessoas próximas, assim como tenha acesso a serviços públicos e comunitários $^{(7,27)}$.

\section{CONSIDERAÇÕES FINAIS}

Os resultados explicitaram que o enfermeiro compreende a juventude e as condições inadequadas da saúde materna como situações adversas ao desenvolvimento infantil. E relata a expressão de processos individuais que podem ser apreendidos como uma manifestação do processo saúde-doença da família, que expõe as suas limitações no cuidado à criança, e a necessidade de uma sociedade inclusiva e igualitária que busque compartilhar seus recursos para o bem-estar de todos os cidadãos. Estas demandas evidenciam que ainda existem limitações na estrutura social e estatal para proporcionar às famílias as condições necessárias para a proteção e cuidado à criança.

Os dados assinalam a necessidade de o enfermeiro aperfeiçoar suas tecnologias de cuidado que promovam a saúde das gestantes adolescentes, incentivem apropriadamente a alimentação infantil e articulem uma adequada rede social de apoio às mães que se encontram em situação de adoecimento com vistas a tentar reduzir a vulnerabilidade no desenvolvimento infantil. A compreensão da vulnerabilidade no cotidiano profissional do enfermeiro e da equipe de Saúde no cuidado à criança permite a reorientação do modelo assistencial, que fundamentado nos princípios de integralidade e equidade, seja direcionado às necessidades da criança e sua família.

Contudo, esta pesquisa tem suas restrições de alcance da realidade, considerando que foi elaborada com dados qualitativos, que são evocações subjetivas, e nem sempre refletem a realidade em sua totalidade. Diante disto, reconhece-se a premência da realização de novas pesquisas que busquem o avanço na estruturação do conceito de vulnerabilidade no campo do desenvolvimento infantil.

\section{REFERÊNCIAS}

1. Ribeiro MO, Sigaud CHS, Rezende MA, Veríssimo MLÓR. Desenvolvimento infantil: a criança nas diferentes etapas de sua vida. In: Fujimori E, Ohara CVS, organizadoras. Enfermagem e a saúde da criança na atenção básica. Barueri: Manole; 2009. p. 61-90.

2. Shonkoff JP, Wood DL, Dobbins MI, Earls MF, Garner AS, McGuinn L et al. The Lifelong Effects of Early Childhood Adversity and Toxic Stress. Pediatrics 2012;129(1):232-46.

3. Brazelton TB, Greenspan SI. As necessidades essenciais das crianças: o que toda criança precisa para crescer, aprender e se desenvolver. Porto Alegre: Artmed; 2002.

4. Shonkoff JP. Protecting brains, not simply stimulating minds. Science. 2011;333(6045):982-3.

5. Shonkoff JP. Levitt P. Neuroscience and the Future of Early Childhood Policy: Moving from Why to What and How. Neuron. 2010;67(5):689-91.

6. Mustard JF. Free market capitalism, social accountability and equity in early human (child) development. Paediatr. child health. 2008;13(10):839-42.

7. Mazza VA, Chiesa AM. Family needs on child development in the light of health promotion. OBJN. [Internet] 2008;7(3) [acesso em 12 out 2012] Disponível: http://www.objnursing.uff.br/index.php/ nursing/article/view/j.1676-4285.2008.1852.

8. Pedroso MLR, Motta MGC. A compreensão das vulnerabilidades socioeconômicas no cenário da assistência de Enfermagem pediátrica. Rev. Gaúcha Enferm 2010;31(2):218-45.

9. Silva DI, Chiesa AM, Veríssimo MLOR, Mazza VA. Vulnerability of children in adverse situations to their development: proposed analytical matrix. Rev Esc Enferm USP. [Internet] 2013;47(6) [acesso em $10 \mathrm{fev}$ 2014] Disponível: http://dx.doi.org/10.1590/S0080623420130000600021

10. Nichiata LYI, Bertolozzi MR, Takahashi RF. The use of the "vulnerability" concept in the Nursing area. Rev. Latino-Am. Enfermagem 2008;16(5):923-8. 
11. Sieving RE, Resnick MD, Garwick AW, Bearinger LH, Beckman KJ, Oliphant JA, et al. A Clinic-Based, Youth Development Approach to Teen Pregnancy Prevention. Am. J. health behav. 2011;35(3):346-58.

12. Wendland J, Levandowski DC. Parentalité à I'adolescence : déterminants et consequences. L'évolution psychiatrique. No prelo 2012.

13. Goodman SH, Tully E, Connell AM, Hartman CL, Huh M. Measuring Children's Perceptions of Their Mother's Depression: The Children's Perceptions of Others' Depression Scale - Mother Version. J Fam Psychol. 2011;25(2):163-73.

14. Silva DI. Vulnerabilidade no desenvolvimento da criança segundo o enfermeiro da Estratégia Saúde da Família. [Dissertação]. Curitiba (PR): Universidade Federal do Paraná; 2012.

15. Curitiba (PR). Secretaria Municipal da Saúde. Plano Municipal deSaúde deCuritiba:2010-2013. Curitiba; 2010.

16. Nery IS, Mendonça RCM, Gomes IS, Fernandes ACN, Oliveira DC. Reincidência da gravidez em adolescentes de Teresina, PI, Brasil. Rev. bras. enferm. 2011;64(1):31-7.

17. Soejima CS, Weber LND. O que leva uma mãe a abandonar um filho? Aletheia. 2008;28:174-87.

18. Sackoff JE, Yunzal-Butler C. Understanding Causal Pathways: Response to "Teen Pregnancy and the Achievement Gap Among Urban Minority Youth". J. sch. health 2012;82(7):301-2.

19. Ladomenou F, Kafatos A, Galanakis E. Risk factors related to intention to breastfeed, early weaning and suboptimal duration of breastfeeding. Acta Paediatr. 2007;96(10):1441-4.

20. Baxter J, Cooklin AR, Smith J. Which mothers wean their babies prematurely from full breastfeeding? An Australian cohort study. Acta Paediatr. 2009;98(8):1274-7.

21. Ponce de Leon CGRM, Funghetto SS, Rodrigues JCT, Souza RG. Vivência da amamentação por mães-adolescentes. Cogitare enferm [Internet] 2009;14(3):540-6. [acesso em 15 fev 2014] Disponível: http://ojs.c3sl.ufpr.br/ojs2/index.php/cogitare/article/ view/16187/10705

22. Wright CM, Parkinson KN. Drewett RF. Why are babies weaned early? Data from a prospective population based cohort study. Arch. Dis. Child. 2004;89(9):813-6.

23. Sultan AN, Zuberi RW. Late weaning: The most significant risk factor in the development of iron deficiency anemia at 1-2 years of age. J Ayub Med Coll Abbottabad. 2003;15(2):3-7. perceived social support as predictors of cognitive function trajectories during the first 3 years of life for preterm infants in Wisconsin. Child Care Health Dev. 2012;38(3):425-34.

25. Lee T, Holditch-Davis D, Shandor MM. The influence of maternal and child characteristics and paternal support on interactions of mothers and their medically fragile infants. Res Nurs Health. 2007;30(1):17-30.

26. Rodrigues GA, Teixeira RCP. A falta de limites na relação pais e filhos e o papel da escola. Rev. Graduação 2011;4(2):26-44.

27. Alexandre AMC, Labronici LM, Maftum MA, Mazza VA. Map of the family social support network for the promotion of child development. Rev Esc Enferm USP. 2012;46(2):272-9.

24. McManus BM, Poehlmann J. Maternal depression and 